\title{
Formal and Informal in Balanced System Specifications
}

\author{
P. Dini, D. Ramazani, G. v. Bochmann \\ University of Montreal, CP 6128, Succ. Centre-Ville, Computer Science \\ and Operational Research Department, Montreal, H3C 3J7, Canada \\ phone: (514) 343-6111, fax: (514) 343-5834, e-mails: \{dini, ramazani, \\ bochmann\}@iro.umontreal.ca
}

\begin{abstract}
The paper presents the relation between balanced system specification by a formal or informal documentation. The text is a technical description of experienced variants, rather than pretentious advices on this topics. We claim that the advent of the object-oriented approach, combined with a mix, formal and informal documentation, in the context of automation, represents a possible solution for either the easiness of knowledge transfer, the use of advanced tools for the system design, or a rapid adaptation from large size enterprises to small ones because of the modularity and reuse. Our report focuses first, on existing techniques, object-oriented methodologies, and second, on lessons learned and retained with respect to the system documentation.
\end{abstract}

Keywords

Models, formalism, documentation, object-oriented, design methods, experiences

\section{INTRODUCTION}

The design of complex systems is one of the most difficult human endeavour, and sometimes it is difficult to directly transfer experiences from a country to another, and even from an enterprise to another. Even further, human experience differs from a small to a medium, or large size enterprise. And the financial support also differs.

On the other hand, the automation of industrial processes or the system design seems to be a right way to enhance the productivity, the quality of service, and the correctness of industrial systems. CAD/CAM systems are the best example of this approach. Also, in the area of telecommunication systems, the automation is inevitable because of increasing tasks and their complexity for a human operator. Automation is almost always accompanied by standards and formalisms for the system representation. Formal specification requires staff with high level abilities which is not possible in all kind of enterprise because of expenses engendered. How to marry our needs with the resources we have, in the context of a continuous evolution of concepts, technologies, and approaches? 


\section{Concept evolution versus industrial needs}

The marriage between the field of computer science (CS) and information system (IS) creates a lot of contradictions regarding fundamental concept definitions. The advent of the object-oriented $(O O)$ approach having the object as a basic paradigm has generated the proposal of many $0 O$ methodologies. We consider that an inadequate formalization of basic constructs reflects that the progress is not finished. Consequently, designers of manufacturing systems need continuously to make trade-offs between the requirements resulting from the mismatch of conceptual evolutions and the industrial reality.

Since formal specification is a necessary condition for automation in the area of distributed communications systems (DCSs), we concentrate in this paper on our experience in using formal and informal specifications for the system description into the object-oriented $(\mathrm{OO})$ approach. We report our experience with respect to the specification of such complex systems. Relevant techniques and methods are briefly presented. Finally, we claim that a certain trade-off between formal versus informal specification must be adopted because the costs spent to re-convert the personnel by training, the time required for a real expertise to be achieved, and system implementations become important.

\section{Experience framework}

The present reported experience has been achieved as members of the project IGLOO (Ingénierie du Génie Logiciel Orienté Objet) which is an ongoing research collaboration between the Computer Research Institute of Montreal, three universities, and six industries. While most research in the past has been done in relation with object-oriented programming in the context of sequential programming languages, the objective of the IGLOO research project (IGLOO, 1992) is to advance the state of the art in the earlier stages of the development cycle, related to requirements analysis and specification development. Special attention is given to the modeling of active objects' behaviors and concurrence, aspects that are important for the specification of reactive systems and real-time applications.

\section{Paper outline}

The remainder of this paper covers several aspects as following. In Section 2 we consider major paradigms of the system development such as automation, formalism, and $O O$ approach. Section 3 reports our experience with respect to several $O O$ methodologies and formal modelling techniques used by them. Finally, Section 4 contains what we have learned and what we recommend with respect to the system documentation.

\section{AUTOMATION PREMISES}

\subsection{Management automation, a fashion?}

Usually, the DCS management is manually accomplished by a human operator at a management centre (for the case of the centralized management), or by many operators using their own management stations (for the case of a distributed platform). Evolutionary changes, usually called dynamic reconfiguration, can be automatically applied in response to failures (sometimes, for unexpected events), to on demand events (changes desired by the human operator, or by system components), or by prevention (in order to avoid undesired situations, or to prevent a rapid degra- 
dation). Methods using predictive mechanisms must be implemented in order to avoid bottlenecks, and to adapt parameters' values.

Coping with these aspects, the automatic management consists of three major tasks namely, (i) the acquisition and the representation of data with respect to the state of the system, (ii) the interpretation of these data having as results appropriate decisions of distributed computing systems (DCSs) state changes, and (iii) the application of these decisions (reconfiguration) and the evaluation of the quality of reconfiguration.

Many studies have identified the automatic management paradigm as having a major importance in DCSs, coping with the complexity and the diversity of several management aspects such as management architectures, data updating techniques, reasoning policies, reconfiguration techniques, as well as methods to evaluate the need and the quality of reconfiguration (Race, 1991)(Stalling, 1993)(Bapat, 1994).

\subsection{Formalism, a need?}

In the last decade, more and more authors emphasize the major role of the formalism in software development. Some consider that a correct specification is formal (Kilov, 1994). Coleman argues that if formal notations were incorporated into object-oriented methods, then we could expect coherent models, rigorous object-oriented methods, and CASE tools which can check the semantic of models (PANEL, 1991).

There are four significant formal approaches for the DCS's specification namely, modular configuration languages, formal description techniques, axiomatic languages, and object-oriented concepts. The standardization organizations have adopted so-called formal description techniques (e.g., LOTOS, Estelle, SDL) for the description of OSI protocols and services, each of them having certain advantages. The axiomatic languages $(\mathrm{Z}$, Object- $\mathrm{Z})$ are extensively analyzed in (Stepney, 1992). The object-oriented approach is a new modelling approach where entities's services are presented into so called «object interfaces».This approach is taken for the information architecture by the international organizations of standardization.

\subsection{0 approach, a mode?}

Current object-oriented (OO) software techniques based on the fundamental paradigm of object have been extensively presented in (Snyder, 1993)(Bochmann et al, 1994). The OO approach must cope with the complexity of DCSs and solve different aspects related to 1) cooperating/ coordinated/communicating systems, (2) reactive/interactive/reflective systems, (3) centralized/ distributed systems, and (4) deterministic/nondeterministic systems. In using OO concepts for DCSs, we distinguish two distinct goals namely, (1) the structural and functional OO models, and (2) the management $\mathrm{OO}$ model.

Each software or hardware DCS entity is modelled as an object which encapsulates data as attribute values and the behavior as methods implementing its own services (operations). Objects communicate via messages with other objects, which means that an object requests to another object to execute one or several of its own operations.

\section{EXPERIENCED METHODS}

We describe here several experienced solutions with respect to existing 00 methodologies, frequently used formalisms, and documentation of complex systems. 


\subsection{Existing 00 methodologies}

Since detailed comparative studies have been presented in the last three years, we only illustrate major specific features of the most used object-oriented methodologies.

- Shlaer-Mellor's method. In this method (Shlaer and Mellor, 1988, 1992) objects are involved in relationships and constructions (subtypes/supertypes or associative objects). The behavioral view is concerned with the Object Communication Model (OCM). Each class is given a state diagram which describes its life-cycle (state model). State models are expressed in state-transition diagrams (Moore model) and tables. The dynamics of relationships captured by state-transition models, relationships without life-cycles, concurrency, and monitors must be analyzed. Action data flow diagrams (ADFDs) depict the Process Model.

- Wirfs-Brock et al's method. This method covers both the analysis and design phases, since no structured input is required (Wirfs et al, 1990). The analysis phase refers to factoring common responsibilities in order to build class hierarchies, streamlining the collaboration between objects by nesting same encapsulation levels or by creating subsystems. The design phase consists of the definitive collaboration establishing, based on interaction of kind of client/server paradigm.

- Coleman et al's Fusion method. The analysis phase of the Fusion methodology separate the behavior of a system into a user perspective (Coleman et al, 1994). The analysis captures different aspects within two models namely, Object Model which defines the static structure of the information, and Interface Model which specifies the input and output of the system. A System Object Model is derived from the Object Model as a subset of objects relating to the system to be built.

- Booch's method. Specification is build within two logical models (Class, Objects), and two physical models (Module, Process) each of them having in tum a static or dynamic semantic. This permits to separate independent types of analysis and design (Booch, 1994). Statics refers to individual objects and the relationships between classes and modules, while events occurring dynamically can be expressed for classes in the State Transitions Diagrams and for objects in Scenario Diagrams. The inter-object behavior is set out in the object scenario diagrams.

- Rumbaugh et al's OMT method. The methodology uses three kinds of models to describe a system (Rumbaugh et al, 1991). The Object Model defines the objects in the system and their relationships, while object interactions are specified in the Dynamic Model. Data transformations are represented in the Functional Model. Rumbaugh's OMT uses three techniques namely, Object Diagrams for the Object Modeling into a data-perspective, Harel's Statecharts for the Dynamic Model into a behavioral perspective, and Data Flow Diagrams in the Functional Model.

\subsection{Formal modeling techniques used by methodologies}

Several modeling techniques used in software design methods have been experimented and analyzed. Some of them are recommended by the previous $\mathrm{OO}$ developing methodologies.

- The entity-relationship $(E / R)$ approach is a widely accepted technique for data modeling (Chen, 1976). Many OO methods apply the E/R model for identifying objects and their relationships. We consider that this model can be used to easily separate objects (subsystems) from their environments and to discriminate between system internal relations and system-environment relations. A dialect called Object Diagrams is frequently used by different $\mathrm{OO}$ methodologies. 
- The $F S M$ model is a well-known model for describing synchronous sequential machines (Gill, 1962). The model explicitly represents the entire state space. The Communicating Finite State Machines (CFSMs) could be used for describing cooperations.

- Petri Nets (PNs) is more general than FSMs. A Petri Net of cooperating objects formally models the information flow. The PNs (Peterson, 1977) allows to hierarchically model a system, both the hardware aspects (control units), and the software ones (resource allocation, deadlock, process coordination, etc.).

- Statecharts additionally permits timed transitions and nested states (Harel, 1987). Statecharts diagram is an adapted tool to describe the behavior for one object since they could accommodate temporal aspects and the state evolution.

- Objectcharts combine an extension of Statecharts with object-oriented analysis and design techniques (Coleman, 1992). Objects have a trace formalization of their behavior, that is, a set of service requests as a triple $(\mathrm{a}, \mathrm{s}, \mathrm{b})$ where $\mathrm{s}$ is the service between the service required (a) and the service provider (b). There is no type definition, but a class is defined as a template for objects belonging to it. Object relations are described in a configuration diagram.

- Message Sequence Charts refer to the systems specifications with respect to the behavior. Z.120 by WPX/3 (Ccitt, 1993) has standardized MSCs that correspond to information flow diagrams.The object behavior is represented by state transition graphs, whereas block interactions by interaction diagrams accompanied by structured English text.

- Traces are a technique invented by Parnas and Bartussek (1978). An object's externally observable behavior is completely defined if its output values are defined for any trace. We experienced that traces are useful to build scenarios at the border between a system and its environment (Bochmann, 1994).

- Temporal logic (Pnueli, 1977) is suitable for specifying properties on each level of abstraction (requirements), for formal reasoning, and verification. Different non-traditional temporal logic have been proposed, in order to produce precise and complete formal specifications and to improve the understanding. Our experience demonstrates that temporal logic is a useful tool for specifying interactions between objects (subsystems). However, the link of specification with the implementation is more difficult to be established.

\section{WHAT HAVE WE LEARNED?}

\subsection{Development process}

Object-oriented computing is a developmental approach which encompasses a number of concepts whose goal is to produce in a relatively short time software which is more reliable and easier to maintain. It covers all the steps of software development, from analysis to programming. An important feature of a such development approach is that the analysis, design and implementation phases adopt a similar model. The approach permits to model system structures which consists of objects and their relationships, the dynamics of systems (behavioral interaction among these objects), and to impose rules of cooperations (assumptions on invariants, preconditions, or postconditions). Software development tasks are distributed in models corresponding to the appropriate phases. At the analysis phase the analysis model prescribes what is the system and its environment. The design model adds to the analysis model aspects defining how the system operations are performed by object behaviors or inter-objects behaviors. Since machines commonly recognize only programming languages, the design model is converted into an implementation 
model. Consequently, the generality of concepts across software development cycle is embedded into a particular and restrictive model expressed in a formal code (language primitive) and in an informal (structured or non-structured) English text. Ideally, similar to the $\mathrm{OO}$ model, an unified and runable specification is desired, in order to avoid the manual transition between the design model and the implementation model. By experience, an $\mathrm{OO}$ design model does not fit well into a non-OO implementation model. But, and this is a thomy aspect, even into an $\mathrm{OO}$ language this translation is not easy and, more important, it is difficult to re-trace all correspondences between these two models. The problem could be solved across well identified translation relations which we briefly represent in Figure 1. The programer performs an horizontal translation from informal specification to formal specification according to the power of the implementation language and its experience (at least for the structured English text). Non-structured text is hopefully formalized by code components connections. The remainder is classified as code annotations. We remark that this translation is language-dependent and programer-dependent.

\begin{tabular}{|llc|}
\hline phase & formal OO specification & informal specification \\
\hline $\begin{array}{l}\text { design } \\
\text { specification }\end{array}$ & OO-FDTs, Object-Z & $\begin{array}{l}\text { structured English } \\
\text { non-structured English }\end{array}$ \\
\hline $\begin{array}{l}\text { implementation } \\
\text { specification }\end{array}$ & $\mathrm{C}^{++}$, Smalltalk, Eiffel code & textual annotations \\
\hline
\end{tabular}

Figure 1 Horizontal translation between formal and informal specification

\subsection{System documentation}

For a good specification, the programmer plays an important role. First, there are two programmer classes namely, which easily use formal description techniques and which do not. Commonly, actual software documentation has a formal part and an informal part at any level of the development cycle. Second, part of the design informal documentation could be formalized by means of programming languages, while part of design informal documentation is identified as either a structured or non-structured English text in the implementation documentation (program annotations). We mention that the formal and informal parts at the analysis/design level are not exactly the same with respect to those at the implementation level. The balance is influenced by many objective (o) or subjective(s) factors.

Among these factors there are (1) the magnitude of modeled systems (o), (2) the methods chosen for the description of object-oriented models (o), (3) the power of expression offered by the used description techniques (o I s), (4) the kind of programming language (o), and (5) the ability of the programmer $(0 \mid \mathrm{s})$. Establishing itself these criteria and their nature is an objective or/and subjective decisions:

(1) Criteria for the magnitude depend on the real systems versus a particular specification method, i.e. PNs, FSMs, Traces, Objectcharts are suitable for different sizes of applications;

(2) Despite of some authors claim of generality, an object-oriented method is better for a particular type of systems than others;

(3) Here are some examples of the formalism expressiveness: the PNs formalism offers the possibility to describe the concurrence, while the language $\mathrm{Z}$ does not; 
(4) Only with respect to the inheritance we present two criteria: (a) some programming languages allows the multiple inheritance $(\mathrm{C}++)$, while other do not (Smalltalk), and (b) commonly all $\mathrm{OO}$ programming languages offer the possibility of monotonic inheritance, however, Eiffel permits to specify the selective inheritance.

(5) The programmer is an important player. Its experience, ability with a particular method, language, or techniques can effectively improve the quality of the implementation documentation.

In Figure 2, we present possible translations between non-structured English, structured English or formal specification across the analysis, design and implementation phases. We recommend that at each development phase these three parts of the documentation co-exist.

Transitions between software development phases must be carefully documented, especially when the specification forms are translated (for example, cases B and C). Thus, activities attached to each arrow must be precised into an additional documentation.

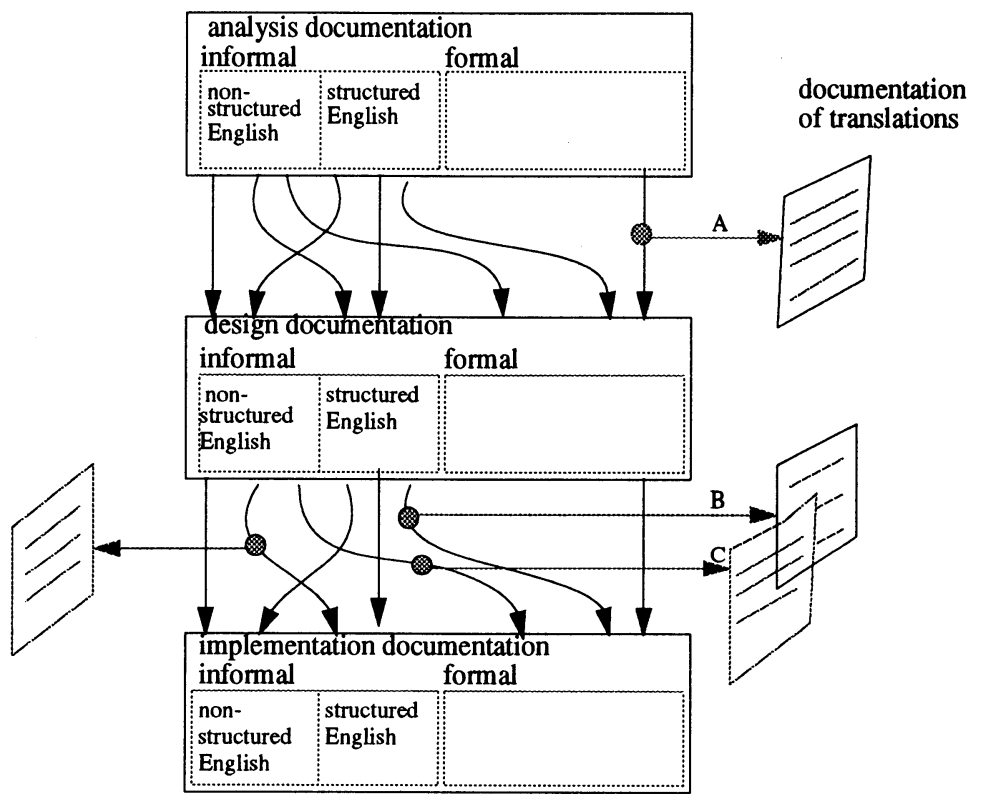

Figure 2 Documentation between phases of development lifecycle

For example, particular procedures describe the $A$ case (from formal to formal) when one passes from analysis to design in the Shlaer-Mellor's method. The authors prescribe transformations rules as procedures for producing architectural objects (from the analysis to design) in the form of specialized class diagrams and class structure charts. From the design to the implementation multiple transformations could be performed, depending on the programmer or on the programming language. In the same method, for example, modules of the archetype class structure charts are expressed as archetype code (code templates) in the chosen implementation language. These tem- 
plates can be filled out from the specialized class structure charts. As presented in Figure 2, this filling is not easy because of various translations (either explicitly or implicitly) when one transits from a development phase to another.

Many structured English texts can be inserted in algorithms (case B). Sometimes this insertion is a part of an implemented method. Sometimes it is together with formal specification, leading the identification of translation more difficult (example, IF < structured English text I formal specification> THEN < structured English text I formal specification>, ELSE <structured English text I formal specification $>$ ). Nevertheless, all it is the program code, but we can distinguish these two forms. Otherwise, part of these structured English texts could be informal annotations of the code. We experienced that it is recommended that the implementation documentation records the reasons of such distinctions. A later interpretation of these documentations could open ways for future adaptations or facilitate them.

Even further, it is possible that a non-structured English text semantics has its formal representation in the code (case $C$ ). Usually, is the programmer which must update the implementation documentation showing unambiguously where, how, and why this approach is used. Commonly, such of situations appear if either there is a superposition between the informal text and the formal specification (implicitly), or the programmer has identified programming language features which can express informal assumptions. Usually, an implementation from a formal specification could contain annotations in structured English derived from those design specifications which are not directly translated into a programming code. Since this derivation is possible, the reverse is not true because first, one could have many derivations, and second, it is difficult to directly isolate within the code a part which is not explicitly indicated by the programmer. Moreover, existing non-structured English text in the design documentation could be textually represented in the implementation documentation in order to ameliorate the implementation comprehension. For example, an Object-Z specification could contain English explanations.

\subsection{What have we retained?}

An ideal solution is not possible, and compromises are inevitable. The best issue is to choose the right level of abstraction in order to easily formalize both the system and its environment. This formalization makes possible the matching between models and their implementations, and facilitates the evolution of the systems by the reuse promotion. An assertional or axiomatic formalization (rules or axioms) facilitates the traceability of development assumptions across the development models, while an operational formalization (state sequences, algorithms) does not in the communicating systems. The drawback is that the former are recommended for small and middle systems, while the latter are specific for large systems. A trade-off is the best solution in order to get advantages from both two forms of formalization.

Evidently, we cannot formalize all. Our opinion is we must first distinguish between critical aspects which will be formalized, from the non-critical aspects, which could be informally done. A structured English for the latter help us to easily understand the code and to make updates without disturbing in an unexpected way the contract between system and its environment.

\section{Acknowledgments}

We would like to acknowledge and thank all colleagues involved in the IGLOO project. Particularly we thank Michel Barbeau, Hafid Mili, Jean-Michel Goutal, and Jean-Marc Serre for fruitful 
discussions, cooperations, and their support. However, the presented opinions are our own experience and do not necessarily reflect the positions of all team members.

\section{REFERENCES}

Bapat, S. (1994) Richer Modeling Semantics for Management Information, Integrated Network Management, III, eds. H.-G. Hegering and Y. Yemini, Elsevier Science Publishers B.V. (North-Holland) 1993, IFIP, pp. 15-28

Bartushek, W., Parnas, D.L. (1978) Using Assertions about Traces to Write Abstract Specifications for Software Engineering, Proceedings of the Second Conf. European Cooperation in Informatics, New York, Springer-Verlag, 1978

Bochmann, v. G., Dini, P., Barbeau, M., Colagrosso, P., Keller, R., Ramazani, D., Somé, S. (1994) Common Concepts for Object-Oriented Analysis and Design, IGLOO Project, Deliverable M.a.1.2., CRIM, January 1994

Booch, G. (1994) Object-Oriented Analysis and Design, The Benjamin/Cummings Publishing Company, Inc.

Ccitt (1993) CCITT Recommendation Z.120, Message Sequence Chart, Geneva, 1993

Chen, P.P. (1976) The Entity-Relationship Model: Toward a Unified View of Data, ACM Transaction on DataBase Systems, vol. 1, no. 1, 1976

Coleman, D., Arnold, P., Bodoff, S., Dollin, C., Gilchrist, H., Hayes, F., Jeremaes, P. (1994) Object-Oriented Development: The Fusion Method, Prentice Hall, Englewood Cliffs

Gill, A. (1962) Introduction to the Theory of Finite State Machines, McGraw-Hill

Harel, D. (1987) Statecharts: A visual formalism for complex systems, Science of Computer Programming no. 8,1987, pp. 231-274

IGLOO (1992)*** Object-oriented Specification and Design Methodologies, Research Proposal, CRIM, March, 1992

Kilov, H., Ross, J. (1994) Information Modeling, PTR Prentice Hall, Englewood Cliffs

PANEL (1991), Formal Techniques for OO Software Development, OOPSLA'91

Pnueli, A. (1977) The Temporal Logic of Programs, 19-th Annual Symposium on Foundations of Computer Science, Providence, RI (IEEE, 1977), pp. 46-57

Race (1991) CFS M200-M210, RACE Common Functional Specifications, Document 8, Network

Management Evolution, Issue B (Research and development in Advanced Communications technologies, in Europe), 1991

Rumbaugh, J., Blaha, M., Premerlani, W., Eddy, F., and Lorensen, W. (1991) Object-Oriented Modeling and Design, Prentice-Hall, Inc.

Shlaer S., Mellor, J.S. (1988) Object-Oriented Systems Analysis: Modeling the World in Data, Yourdon Press, PTR Prentice Hall, Englewood Cliffs

Shlaer S., Mellor, J.S. (1991) Object Life Cycles: Modeling the World in States, Yourdon Press, PTR Prentice Hall, Englewood Cliffs

Snyder, A. (1991) The Essence of Objects: Common Concepts and Terminology, Software and Systems Laboratory, HPL-91-50, June

Stalling, W. (1993) SNMP, SNMPv2, and CMIP: The Practical Guide to Network Management Standards, Addison Wesley Publishing Company

Stepney, S. (1992) Object Orientation in Z, Logica Cambridge Limited, U.K. 
Wirfs-Brock, R.J., Wilkerson, B., Wiener, L. (1990) Designing Object-Oriented Software, Prentice Hall, Englewood Cliffs

\section{BIOGRAPHY}

Petre DINI received the MS in Computer and Automation Science from the Polytechnical Institute of Timisoara, Romania, in 1976. Since, he has developed industrial systems related to CAD/ CAM, robotics, and automatic machine tools. He has interested on knowledge representation in electronics, electrical and mechanical domains, modelled and designed distributed industrial chains, having many publications in these areas. He is currently involved in Ph.D. program at the University of Montreal. His interests are related to automatic reconfiguration in networks and open distributed systems, QoS in multimedia applications, software engineering formalization, software and hardware tests, telecommunications, and the object-oriented concepts related to these fields.

Dunia RAMAZANI received the MS in Computer Science from the University of Montreal, Canada, in 1992. He was a visiting researcher at the Bavarian research Center for Knowledge Based Systems (FORWISS), Munich, Germany, during the first quarter of 1993. There, he has worked on the design and implementation of Object-oriented database management systems. Since then, he has been working towards the Ph. D. at the University of Montreal. As part of his Ph. D. work, he is involved in the IGLOO project, a project aimed at contributing to the advancement of the object paradigm. His current research interests include formal methods, object-oriented software engineering and telecommunication systems.

Gregor v. BOCHMANN (M'82-MS'85) received the Diploma degree in physics from the University of Munich, Munich, West Germany, in 1968 and the Ph.D degree from McGill university, Montreal, P.Q., Canada, in 1971. He has worked in the area of programming languages, compiler design, communication protocols, and software engineering and has published many papers in this area. He holds the Hewlett-Packard-NSERC-CITI chair of industrial research on communication protocols in the Département d'informatique et de recherche opérationnelle, Université de Montréal, Montréal. His present work is aimed at design methods for communication protocols and distributed systems. He has be involved in the standardization of formal description techniques for OSI. From 1977 to 1978 he was a Visiting Professor at the Ecole Polytechnique Fédérale, Lausanne, Switzerland. From 1979 to 1980 he was a Visiting Professor in the Computer Systems Laboratory, Stanford University, Stanford, C.A. From 1986 to 1987 he was a Visiting Researcher at Siemens, Munich. He is presently one of the scientific directors of the Centre de Recherche Informatique de Montreal (CRIM). 